\title{
Equal or not? Women hold less prestigious roles at respiratory medicine conferences than men
}

\author{
To the Editor:
}

The gender imbalance in medicine has been a topic of increasing interest and discussion. There are increasing proportions of graduating female medical students globally, with $41.1 \%$ of Australian doctors, $47 \%$ of UK doctors and $46 \%$ of medical residents in the USA being female $[1,2]$. However, in positions of influence, the proportions change, such that in the USA only $21 \%$ of medical professors and $16 \%$ of medical deans are female [3]. Postulated reasons for this include implicit and unconscious gender bias and fewer promotion opportunities available to women.

Similarly, in Australia, across "STEM" (science, technology, engineering and mathematics) disciplines, women comprise $55 \%$ of bachelor degree matriculates in sciences and mathematics, and $46 \%$ of $\mathrm{PhD}$ students; however, this translates to only $20 \%$ of senior professors being female [4]. Attempts to correct this gap in medicine have been made, including the Athena SWAN Charter, enacted in the UK in 2005, to advance the careers of women in medicine, and "SheNote Speaker", a database designed to increase visibility of women in respiratory and cardiovascular research [5], a factor that is key to career progression.

The exact gender balance in respiratory medicine is currently unclear. This study aimed to analyse the current state of gender distribution in research within respiratory medicine.

Using a methodology similar to SLEEMAN et al. [6], one author reviewed information from publicly available online conference programmes regarding attendance and participation at one large international respiratory conference (the European Respiratory Society (ERS) congress in 2017) and two smaller national respiratory meetings (the British Thoracic Society (BTS) winter meeting in 2017, and the Thoracic Society of Australia and New Zealand (TSANZ) annual scientific meeting in 2018). These three conferences were chosen purposely as they attract delegates from different parts of the world and vary considerably in size, with the ERS congress receiving >22000 delegates in recent years, whereas the BTS and TSANZ meetings attract a few hundred each year. The genders of participants assuming the roles of invited speaker at sessions or symposia, session or symposium chairperson, and first author or last author for oral and poster presentations, were compared. Gender was determined by the participant's name and, where this was unclear, a search for the person or the first name was undertaken using internet and research database searches to establish gender.

Data are reported as counts and frequencies. The Chi-squared test was used to examine associations between gender and conference role, with comparisons made between the difference conferences. $\mathrm{p}<0.05$ was taken to indicate statistical significance. Repeat presenters were not accounted for in the tallied results.

Of all participants $(23500)$ at the three conferences, 10689 assumed "active" roles as invited speakers, presenting oral or poster research abstracts (first and last author) or chairing sessions/symposia. For the individual conferences there were 9605 active participants at the ERS congress, 811 at the TSANZ annual scientific meeting and 273 at the BTS winter meeting. The pooled results from all three conferences demonstrate that a significantly larger proportion of men compared to women held roles as chairperson and invited speakers (figure 1). For poster and oral presentations, there were significantly more female first authors, with 1713 females and 1562 males ( $p=0.008)$. However, significantly more men (2057 males and

@ERSpublications

Analysis of the sex distribution of presenters in national and international conferences in 2017 demonstrates a significantly lower proportion of women in more prominent roles compared with men http://bit.ly/2lGJz02

Cite this article as: Raviskanthan M, Rees M, Douglass J, et al. Equal or not? Women hold less prestigious roles at respiratory medicine conferences than men. Eur Respir J 2020; 55: 1900701 [https://doi.org/ 10.1183/13993003.00701-2019]. 


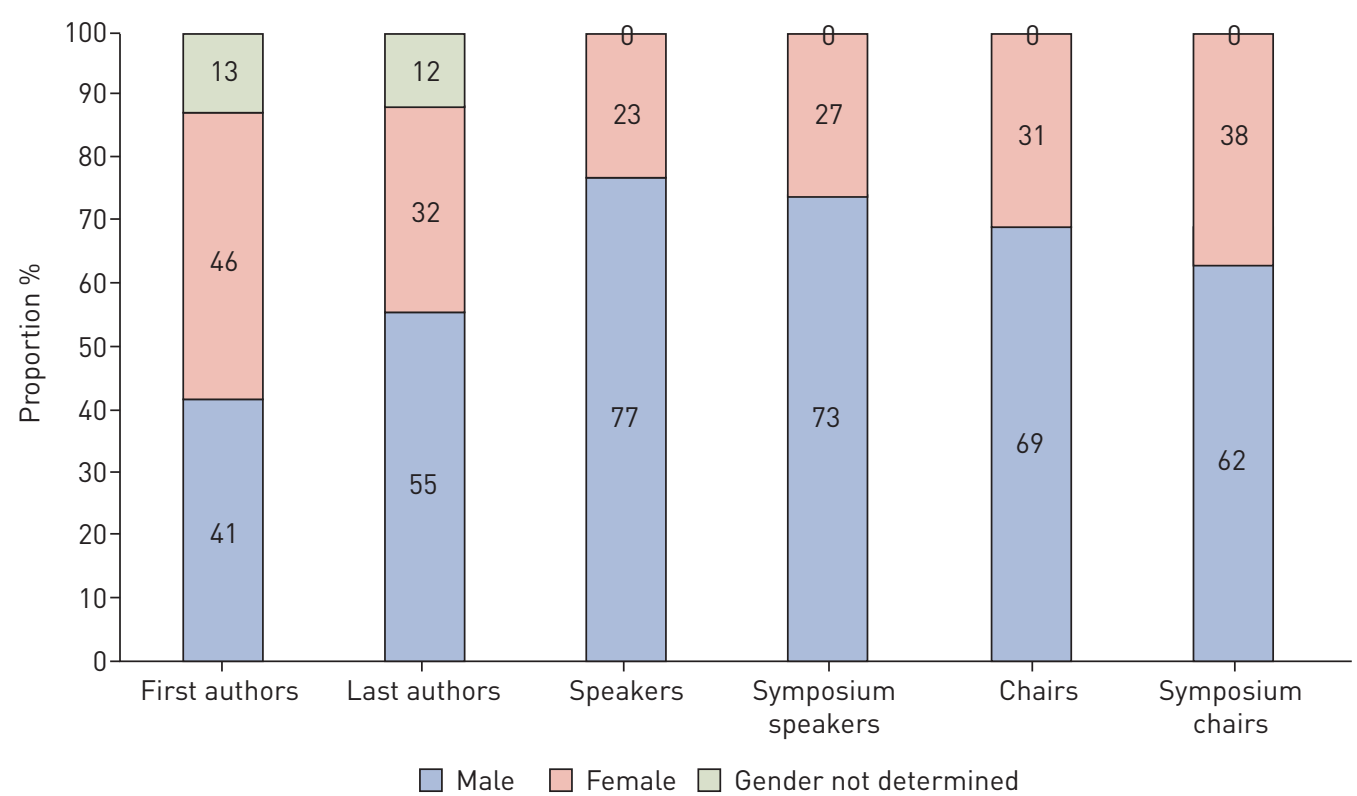

FIGURE 1 Overall gender proportions characterised by role in conference (pooled results for the three conferences).

1202 females, $\mathrm{p}<0.001$ ) were listed as last authors on presentations, with this position representing the most senior member of the research team.

Compared to the other two conferences, at the ERS congress there was the greatest gender imbalance for participants assuming the roles of invited speaker $(23 \%$ female, $\mathrm{n}=225 ; \mathrm{p}<0.001)$ or session chairperson ( $31 \%$ female, $\mathrm{n}=367 ; \mathrm{p}<0.001)$. At the BTS winter meeting, fewer invited speakers were female $(35 \%, \mathrm{n}=34$; $\mathrm{p}=0.004)$; however, similar numbers of males and females were invited to chair sessions ( $45 \%$ female, $\mathrm{n}=76 ; \mathrm{p}=0.19$ ). At the TSANZ annual scientific meeting, similar numbers of males and females were invited to speak ( $49 \%$ female, $n=297 ; \mathrm{p}=0.8)$ and to chair sessions $(46 \%, \mathrm{n}=76 ; \mathrm{p}=0.2)$.

Of those participants with more than five conference presentations or roles, only one-third were female ( 200 out of $605, \mathrm{p}<0.005$ ). Similarly, only $20 \%$ of participants with $>10$ presentations or roles were female (21 out of $105, \mathrm{p}<0.005)$.

Gender could not be determined by name for $9 \%$ of the active participants at the three conferences. However, a sensitivity analysis, in which it was assumed that all the missing names were women, did not significantly alter the study findings.

At the entry level of academic research (first author conference presentations) in respiratory medicine, women participate significantly more than men. However, a gender gap appears and widens significantly as the conference role becomes more senior and prestigious, with fewer women involved. Importantly these findings are not isolated to respiratory medicine. A similar analysis examining the roles of women at the European Association of Palliative Care conferences in the preceding 5 years demonstrated that while more women $(84 \%)$ were speakers at free communication sessions, only $26 \%$ of invited plenary speakers (a more prestigious role) were female [6].

Notably, in this study there was a clear difference in women's participation between the three conferences. While anecdotally it has been suggested that women may be more reluctant to travel an increased distance to actively participate in an international conference such as ERS, it should be noted that delegates attending the TSANZ conference, which is held in Australia or New Zealand, regularly travel vast distances ( $\geqslant 4 \mathrm{~h}$ by aeroplane to fly from east to west Australia alone), and this does not affect female participation. Furthermore, there is no current evidence suggesting that women in respiratory medicine are more anxious than men regarding presenting at international conferences or using a foreign language.

Alternatively, the policies of each specialist society (ERS, BTS and TSANZ) regarding gender balance at conferences may partially explain the differences in female participation between the three conferences, although these were not publicly available. Notably, freely available online data suggests that both the ERS and TSANZ have strong active women in leadership positions. Six (46\%) out of the 13 TSANZ board members are female, and currently five out of seven branch presidents are female. Similarly, within the ERS, the board comprises six women and eight men, and the current president-elect is female. While the 
TSANZ has a specific policy of gender balance for invited plenary speakers, it is not known whether the ERS and BTS have similar policies. Importantly, these results would suggest that both stringent policies advocating for gender balance in conference leadership roles, and women holding leadership positions within each society, are extremely important to close the current gender gap.

Importantly, gender imbalance is not simply an issue related to conference programming or specialist societies' policies. The causes for the disparity between male and female in both specialist medical practice and academic medicine are multifactorial. Previous studies have determined that women tend to have a greater interest in teaching than research, and that the gender bias and lack of adequate mentorship contributes to the disparity of genders in academic medicine [7]. Surveys of medical students demonstrate that female students have lower interest in undertaking research compared with male counterparts [8]. In addition, bullying and sexual harassment have been identified as important issues in both clinical and academic medicine, and present barriers to career advancement for women [9].

Programmes such as the Athena SWAN scheme have been successful. Enacted in 2005, the programme acts as an award system to educational institutions who demonstrate gender equity. It has increased support for women's careers, provided mentoring and increased access to funding and has improved visibility of women in academia. However, the ability of this programme alone to change intrinsic bias and the culture of scientific medicine has been questioned [10], with a persistence of the gender bias in the UK after 12 years of this programme, and concerns regarding ongoing pay gaps and power imbalances. It is likely that institutional and societal changes are also needed, to provide support for women in childcare and other duties, and to further address gender pay gaps, in addition to support in research.

Targets moving forward will require a greater understanding of why this disparity is ongoing, the impact of culture and gender bias, and its contributory factors. Globally, support is needed for women to advance their careers, through sponsorship programmes, access to funding and incentivising the return to academia post-maternity leave. Conference organisers could consider enforcing gender ratios amongst invited chairs and speakers at events. Bullying and sexual harassment must be penalised appropriately, and appropriate reporting systems should be instituted. Promoting academic medicine to women should start early, from medical school, and mentors and sponsorship should be instituted.

Importantly, the data obtained in this study provides a fixed time point analysis of the current state of gender balance in academic respiratory medicine at conferences at the national and international level in the 2017-2018 period, therefore cannot be used to assess trends of change. However, trend data will be important in the future to determine whether any active interventions have been effective. In addition, the differences in sizes of conferences results in the tallied figures being heavily skewed by the findings of the ERS conference.

The gender gap in academic respiratory medicine is evident at national and international conferences. Without fostering academia and maintaining excellence in all clinicians, we are compromising our focus on evidence-based medicine. Further research to monitor the efficacy of current programmes that aim to support women and implementation of new programmes is required to ensure that all doctors, regardless of gender, have equal opportunities in career progression.

Mayurapriya Raviskanthan ${ }^{1}$, Megan Rees $^{2}$, Jo Douglass ${ }^{3}$, Katherine Sleeman ${ }^{4}$, Irene Higginson $\oplus^{5}$ and Natasha Smallwood $\oplus^{6,7}$

${ }^{1}$ Medicine, Royal Melbourne Hospital, Parkville, Australia. ${ }^{2}$ Royal Melbourne Hospital, Melbourne, Australia. ${ }^{3}$ Dept of Immunology, The Royal Melbourne Hospital, Melbourne, Australia. ${ }^{4}$ King's College London, Florence Nightingale Faculty of Nursing, Midwifery and Palliative Care, Cicely Saunders Institute, London, UK. ${ }^{5}$ Palliative Care, Policy and Rehabilitation, Cicely Saunders Institute, London, UK. ${ }^{6}$ Dept of Respiratory and Sleep Medicine, Royal Melbourne Hospital, Melbourne, Australia. ${ }^{7}$ Dept of Medicine, Royal Melbourne Hospital, University of Melbourne, Melbourne, Australia.

Correspondence: Dr Mayurapriya Raviskanthan, Dept of Medicine, Royal Melbourne Hospital, Parkville, Melbourne, Australia. E-mail: mayurapriya.raviskanthan@mh.org.au

Received: 06 Apr 2019 | Accepted after revision: 02 Sept 2019

This article has an editorial commentary: https://doi.org/10.1183/13993003.01847-2019

Conflict of interest: M. Raviskanthan has nothing to disclose. M. Rees has nothing to disclose. J. Douglass is an employee of Royal Melbourne Hospital and the private medical practice Melbourne Allergy, Asthma and Immunology Consultants, and holds an honorary appointment at the Universtiy of Melbourne; and reports personal fees for lectures from and participation in trials for AstraZeneca, participation in trials for GlaxoSmithKline, Sanofi, Grifols, BioCryst and Equilium, grants and personal fees for advisory board work and lectures from Novartis, grants and personal fees for advisory board work from CSL-Behring, outside the submitted work. K. Sleeman has nothing to disclose. I. Higginson has nothing to disclose. N. Smallwood reports holding an unpaid role as the president elect of the Victorian Branch of the Thoracic Society of Australia and New Zealand. 


\section{References}

1 Australian Institute of Health and Welfare. Medical Practitioners Workforce 2015. www.aihw.gov.au/reports/ workforce/medical-practitioners-workforce-2015/contents/how-many-medical-practitioners-are-there Date last updated: August 24, 2016.

2 British Medical Association. Health Policy \& Economic Research Unit. 2014 UK Medical Workforce Briefing May 2015. www.agcas.org.uk/write/MediaUploads/Resources/MCAN\%20careers\%20information\%20resources/BMA 2014_UK_Medical_Workforce_Briefing_\%28May_2015\%29.pdf.

3 Association of American Medical Colleges. The State of Women in Academic Medicine 2013-2014. https://store. aamc.org/the-state-of-women-in-academic-medicine-the-pipeline-and-pathways-to-leadership-2013-2014.html.

4 Science in Australia Gender Equity. Gender Equity in STEMM. www.sciencegenderequity.org.au/gender-equity-in-stem/ Date last updated: August 16, 2016.

5 Equality Challenge Unit. Athena SWAN Charter. www.ecu.ac.uk/equality-charters/athena-swan/ Date last accessed: January 8, 2020

6 Sleeman KE, Koffman J, Higginson IJ. Leaky pipeline, gender bias, self-selection or all three? A quantitative analysis of gender balance at an international palliative care research conference. BMJ Support Palliat Care 2019; 9: $146-148$.

7 Edmunds LD, Ovseiko PV, Shepperd S, et al. Why do women choose or reject careers in academic medicine? A narrative review of empirical evidence. Lancet 2016; 388: 2948-2958.

8 Guelich JM, Singer BH, Castro MC, et al. A gender gap in the next generation of physician-scientists: medical student interest and participation in research. J Investig Med 2002; 50: 412-418.

9 Jagsi R, Griffith KA, Jones R, et al. Sexual harassment and discrimination experiences of academic medical faculty. JAMA 2016; 315: 2120-2121.

10 Ovseiko PB, Chapple A, Edmunds LD, et al. Advancing gender equality through the Athena SWAN Charter for Women in Science: an exploratory study of women's and men's perceptions. Health Res Policy Syst 2017; 15: 12. 